\title{
Molecular dynamics study of homogeneous and inhomogeneous phase in charged colloids: The influence of surface charge density
}

\author{
Wenze Ouyang, Hongwei Zhou, Shenghua Xu*, Zhiwei Sun** \\ Key Laboratory of Microgravity (National Microgravity Laboratory), Institute of Mechanics, Chinese Academy of Sciences, Beijing 100190, China
}

\section{H I G H L I G H T S}

- Investigate the dynamic process of crystallization and voids formation in charged colloids.

- Influence of surface charge density on homogeneous to inhomogeneous phase transition.

- Finite size effects on the phase behavior of charged colloids.

- Show that Sogami and Ise theory cannot explain the reentrant transition of highly charged colloidal systems.

\section{A R T I C L E I N F O}

\section{Article history:}

Received 12 July 2013

Received in revised form

10 September 2013

Accepted 1 October 2013

Available online 11 October 2013

\section{Keywords:}

Molecular dynamics

Charged colloids

Crystallization

Voids

\section{G R A P H I C A L A B S T R A C T}

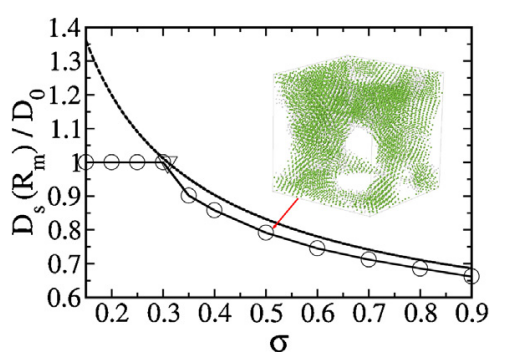

\begin{abstract}
A B S T R A C T
Compared with previous Mote Carlo (MC) simulations with 432 particles, molecular dynamics (MD) simulations with much larger number of particles have been carried out to investigate the dynamic process of the structural ordering and voids formation in charge stabilized colloidal suspensions. Sogami and Ise (SI) potential which has a long-range attraction is used to represent the interaction between colloidal particles. As increasing the surface charge density on the colloidal particles, the data obtained from the simulations, such as the crystallization, bcc-fcc phase transition, homogeneous to inhomogeneous transition and the voids formation, are in agreement with previous observations of MC simulations and experiments. The effects of particle number used in the simulations are studied in detail. MD simulations in highly charged colloidal system with small sizes show very few crystallized particles, in accord with the results of MC simulations. However, the structure in the system with larger number of particles is always the voids coexisting crystallites instead of a glasslike or disordered inhomogeneous phase, indicating that the glasslike or disordered phase region obtained at very high charge density in small system is an artifact produced by very limited number of particles used in the simulations. Therefore, SI potential is not applicable for explaining the reentrant transition of highly charged colloidal systems.
\end{abstract}

(C) 2013 Elsevier B.V. All rights reserved.

\section{Introduction}

Colloidal suspensions have been considered to model condensed matter systems, due to their structural ordering and related phase transitions [1-4]. Among the colloidal studies, suspensions of charged colloidal spheres are ideal for model studies of

\footnotetext{
* Corresponding author. Tel.: +86 82544099; fax: +86 82544096.

** Corresponding author.

E-mail addresses: xush@imech.ac.cn (S. Xu), sunzw@imech.ac.cn (Z. Sun).
}

crystallization because they are easily observed and their forces are readily manipulated by controlling the chemistry of the suspension medium [4-6]. It has been believed for a long time that the interaction between charged colloidal particles can be described by the Derjaguin-Landau-Verwey-Overbeek (DLVO) theory, in which the potential is predominantly screened Coulomb repulsion except the van der Waals attraction at very short distances [7]. For stable suspensions the van der Waals attraction is negligible so that the DLVO potential can be referred to the screened Coulomb repulsion only. DLVO theory has been used to explain some properties of homogeneous phases, e.g., the ordering in charged colloids. However, 
some experimental evidence has emerged such as the nonspace filling localized ordered structures coexisting with disordered regions observed by microscopy and scattering experiments, the existence of stable voids, vapor-liquid condensation and the reentrant phase transition, which suggest the existence of a long-range attraction between the charged colloids [8-14].

In order to explain the above-mentioned experimental results in charged colloids, Sogami and Ise (SI) have proposed an effective interaction model based on the Gibbs free energy of the interaction [15]. This model not only takes into account the large size difference between colloidal particles and small ions, but also relates the variation of macroion charge to the release of counterions. Based on this model, Tata and Ise have performed Monte Carlo (MC) simulations to observe the homogeneous and inhomogeneous structures in charged colloidal systems. Their results of MC simulations seem to be able to successfully explain some experimental observations, e.g. the formation of colloidal crystals, reentrant transitions, and the coexistence of voids with ordered and disordered regions [16-18]. For the past decades, a debate has been going on in colloidal science between the supporters of the classic DLVO theory and those who tend to accept the SI theory [19]. For instance, van Roij et al. used a volume term theory to explain some important feature of the phase diagram of charged suspensions at very low ionic strengths from the first principles, showing that there is no need to invoke longrange attraction between particles [20,21]. Moreover, it is difficult to quantitatively compare the results of previous MC simulations with those of experiments because MC steps are not real time. Until now, there are very few dynamics simulations for the study of inhomogeneous and homogeneous structures in charged colloidal systems with SI potential. On the other hand, the number of colloidal particles used in MC simulations by Tata and Ise is very small (typically $N=432$ ), possibly due to the computational cost. But, many earlier simulations have already shown that the system size has a large effect on the crystallization process [22-25]. Such small number of colloidal particles used in MC simulations may not be appropriate for the observations of structural ordering in the system, although the observed results in several different system sizes (up to $N=1024$ ) are the same within statistical error $[17,18]$.

In this work, we carry out molecular dynamics (MD) simulations in charged colloidal systems. Through simulations with different colloidal parameters, we can obtain inhomogeneous structures such as voids coexisting with ordered regions, and the homogeneous structures such as crystal structure without voids. The dynamic process of crystallization and the formation of voids is investigated in MD simulations. We mainly study the homogeneous and inhomogeneous phase as a function of surface charge density on the colloidal particles, keeping the other parameters fixed. The number of colloidal particles used in MD simulations (typically $N=10,976$ ) is sufficiently large to alleviate finite size effects.

\section{Details of simulation}

The interaction between the charged colloidal particles in the simulation box is SI pair potential [15], which has the following form,

$$
U\left(r_{i j}\right)=B\left(\frac{A}{r_{i j}}-\kappa\right) \exp \left(-\kappa r_{i j}\right)
$$

where $r_{i j}$ is the pair distance between $i$ th and $j$ th particle, the inverse Debye screening length $\kappa$ is given as

$$
\kappa=\sqrt{\frac{e^{2}\left(n_{p} Z+C_{s}\right)}{\epsilon k_{B} T}},
$$

and the parameters

$$
\begin{aligned}
& A=2+\operatorname{coth}\left(\frac{\kappa d}{2}\right) \\
& B=\frac{1}{2 \pi \epsilon}\left[\frac{Z e \sinh (\kappa d / 2)}{\kappa d}\right]^{2} .
\end{aligned}
$$

$Z e$ is the charge on the particle (related to effective charge density by $\left.Z e / \pi d^{2}\right), C_{S}$ is the salt concentration, $\epsilon$ is the dielectric constant of water, and $k_{B}$ is the Boltzmann constant. The temperature $T$ is fixed at $298 \mathrm{~K}$ and the diameter of particles $d$ is taken as $110 \mathrm{~nm}$ in simulations. SI potential has a minimum at the position $R_{m}$,

$$
R_{m}=\frac{A+\sqrt{A(A+4)}}{2 \kappa} .
$$

For convenience, the reduced units are used in the simulation. The basic units are chosen as follows: energy units $k_{B} T$, the mass of colloidal particles $m$, and the relevant length scale $L_{0}=\sqrt[3]{(6 V / \pi N)}$, where $N$ is the total number of particles and $V$ is the volume of simulation box. In such reduced units, we can get $d=\sqrt[3]{\phi}(\phi$ is the volume fraction of colloidal particles), and the average interparticle separation $D_{0}$ of homogeneous body-centered-cubic (bcc) or face-centered-cubic (fcc) crystal is constant $\left(D_{0}=0.879\right.$ for bcc and $D_{0}=0.904$ for fcc, respectively).

The MD simulations are performed in NVT ensemble. The period boundary condition is applied. The simple truncation of interactions and the minimum image convention are used [26,27], where the cutoff distance is chosen as $r_{c u t}=2.0$ in reduced units. The equation of particles' motion is integrated using velocity Verlet algorithm with the time step $\delta t=0.0005$ and the constant temperature is controlled via Berendsen thermostat [28]. Initially we place the particles randomly in a three-dimensional cubic simulation box. If a particle has an overlap with any other particles, it is rejected immediately and a new position will be generated randomly until no overlap happens. After a long enough period of MD time steps (typically $1.9 \times 10^{6} \delta t$ ), the system can reach equilibrium and some parameters of the properties are calculated during another long runs $\left(10^{5} \delta t\right)$, in order for accurate average value obtained.

\section{Results and discussion}

According to Eqs. (1) and (2), the interaction between colloidal particles is determined by many factors, such as the salt concentration, surface charge density and volume fraction of the colloid, etc. Different values of those factors which influence the interactions will result in different phase behaviors of the system. In this research, we keep the diameter and the volume fraction of the colloid fixed, i.e, $d=110 \mathrm{~nm}$ and $\phi=0.03$, while the salt concentration is taken as $C_{S}=0$. Here the parameters chosen are the same to those of previous MC simulations in order for a convenient comparison [17]. Therefore, the research is only focused on the phase behaviors of the system as a function of the charge density $\sigma$. The charge density $\sigma$ on the colloid plays an important role in determining the ordering of the charge stabilized suspensions as it alters the range and strength of the interaction. In the case of polystyrene colloids, a variation in $\sigma$ is possible by controlling the concentration of charge determining salts during synthesis; In the case of charge stabilized silica colloids, $\sigma$ can be varied by controlling the sodium hydroxide concentration [12].

When the charge density $\sigma$ is small, the system exhibits a liquid state. Increasing the value of $\sigma$, colloidal particles begin to crystallize and a freezing transition occurs so that the structure ordering of the system takes place somehow. The colloidal particles' motion will be restricted to local regions after the crystallization is completed. The mean square displacement (MSD) measures the 


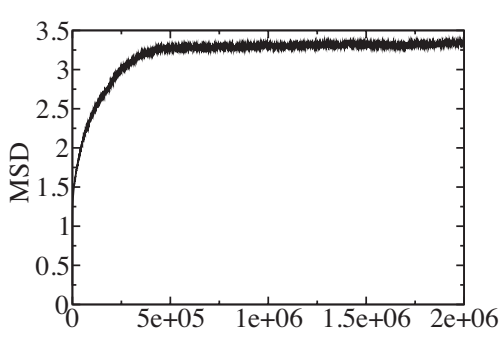

$\delta \mathrm{t}$

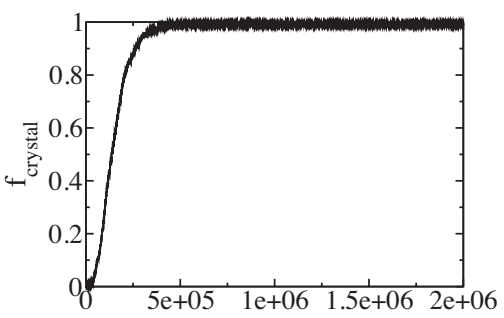

$\delta \mathrm{t}$

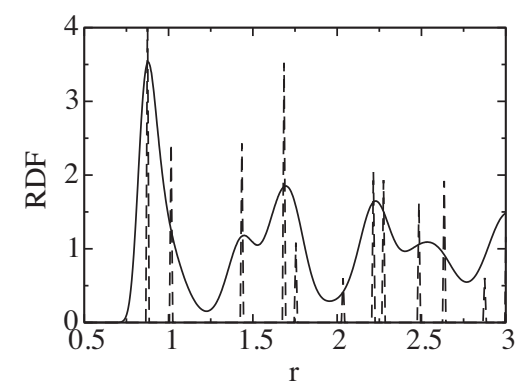

Fig. 1. From top to bottom: mean square distance (MSD) versus time step, the fraction of crystallization versus time step, averaged radial distribution function (RDF) after equilibrium. The dashed lines in RDF curve show the peaks of perfect bcc structure. The charge density is $\sigma=0.2 \mu \mathrm{C} / \mathrm{cm}^{2}$.

distance that the particle in average travels during the simulation, and it can be used to characterize whether the particles' motions are restricted to local regions or not. MSD is calculated by averaging over all the particles in the system and thus is given by

$\operatorname{MSD}=\frac{1}{N} \sum_{i=1}^{N}\left(\mathbf{r}_{i}(t)-\mathbf{r}_{i}(0)\right)^{2}$

The evolution of MSD with the time step is displayed in the top panel of Fig. $1\left(\sigma=0.2 \mu \mathrm{C} / \mathrm{cm}^{2}\right)$. The MSD has an obvious increase since the simulation starts, and then reaches a plateau after about $5 \times 10^{5} \delta t$, which reflects that the system has reached equilibrium and the particles are moving only around their equilibrium positions.

In the simulations, it is needed further to be able to distinguish particles that are part of the crystal from those belong to the liquid. We use the method presented by Frenkel and coworkers [29]. In this method, the type of a particle is determined based on its local environment. First we consider the set of nearest neighbors of a particle $i$ as all particles $j$ that are within a given radius $r_{q}$ from $i$. The value of $r_{q}$ is chosen arbitrarily which is close to the first minimum of radial distribution function (RDF) curve. Then, the complex vector $q_{l m}(i)$ of particle $i$ is defined by

$q_{l m}(i)=\frac{1}{N_{n b}(i)} \sum_{j=1}^{N_{n b}(i)} Y_{l m}\left(\mathbf{r}_{i j}\right)$.

Here $N_{n b}$ is the number of nearest neighbors of particle $i$. The functions $Y_{l m}\left(\mathbf{r}_{i j}\right)$ are the spherical harmonics, $\mathbf{r}_{i j}$ is the vector from particle $i$ to particle $j, l$ is a free integer parameter and $m$ is an integer that runs from $m=-l$ to $m=l$. To identify solid-like particles, the integer parameter $l$ is taken as $l=6$ and $q_{6 m}(i)$ should be normalized

$$
d_{6 m}(i)=\frac{q_{6 m}(i)}{\left[\sum_{m=-6}^{6}\left|q_{6 m}(i)\right|^{2}\right]^{1 / 2}}
$$

Using the normalized complex vectors $d_{6 m}(i)$, a scalar product which measures the correlation between neighboring particles $i$ and $j$ can then be defined by

$S_{i j}=\sum_{m=-6}^{6} d_{6 m}(i) \cdot d_{6 m}^{*}(j)$,

where the * indicates complex conjugation. Two neighboring particles $i$ and $j$ are defined to be connected if the scalar product $S_{i j}$ described above exceeds a given value, typically $S_{i j}>0.7$. A particle will be identified as solid-like if the number of connections is above a certain threshold, typically 8 . Finally, we define the degree of crystallinity $f_{\text {crystal }}$ of a sample as the number of particles in solid-like environments divided by the total number of particles $N$.

In the middle panel of Fig. 1 , the evolution of $f_{\text {crystal }}$ during crystallization is given. Starting from the initial random configuration at which $f_{\text {cryatal }} \approx 0$, more and more colloidal particles begin to crystallize so that $f_{\text {crystal }}$ increases apparently. When the system reaches equilibrium, almost all of the colloidal particles become solid-like which indicates the system is crystallized completely. The RDF can be used to analyze the long-range order of the system and distinguish the structure of the resulting crystals. The bottom panel of Fig. 1 shows the curve of averaged RDF after the system's equilibrium. In the curve of averaged RDF, there are a lot of peaks, the positions of which fit bcc crystal structure. Therefore, we can make the judgement that the crystal structure formed in the system when equilibrium is bcc. Of course, the peaks are not so sharp as RDF curve of perfect bcc structure, because the colloidal particles can always vibrate around the lattice sites when equilibrium. Note that the number of particles $N=10,976$ we choose is favorable for fcc structure, nevertheless the bcc structure is still formed. For having a clear map of the bcc crystallization process, Fig. 2 shows some snapshots of the colloidal configuration during the simulation at $\sigma=0.2 \mu \mathrm{C} / \mathrm{cm}^{2}$. The liquid-like particles are plotted as gray spheres and the solid-like particles are plotted as green spheres.

Upon increasing the charge density $\sigma$ to $0.3 \mu \mathrm{C} / \mathrm{cm}^{2}$, the system exhibits a fcc crystalline order (see Fig. 3). Actually we also performed the simulations at $\sigma=0.25 \mu \mathrm{C} / \mathrm{cm}^{2}$, finding that the system still exhibits a bcc crystalline order. Hence the bcc-fcc transition here is expected to take place between the values of $\sigma=0.25 \mu \mathrm{C} / \mathrm{cm}^{2}$ and $0.3 \mu \mathrm{C} / \mathrm{cm}^{2}$, suggesting $\sigma=0.3 \mu \mathrm{C} / \mathrm{cm}^{2}$ is nearly close to the boundary of bcc-fcc phase transition. Such result above is different from the results of MC simulations [17,18], in which the bcc-fcc transition occurred at larger charge density. The difference is believed to be caused by the finite size effects, although Tata and Ise stated that the number of particles used in simulations is sufficient and the results of simulations are independent of the system size when $N \geq 432$. In order to have a detailed study on the finite size effects, MD simulations with the same number of particles $N=432$ as used in MC simulations have been performed by varying the charge density $\sigma$. The crystal structure is always bcc when $\sigma=0.15-0.35 \mu \mathrm{C} / \mathrm{cm}^{2}$, in agreement with previous MC simulations. This means that both MC and MD simulations with smaller number of particles will make the bcc-fcc boundary shift to larger charge density, indicating a significant finite size effects on the bcc-fcc transition. More details about the finite size effects on the phase behaviors of the system will be discussed in the latter of this paper. Now we turn to the bcc-fcc phase transition. Indeed, it has 


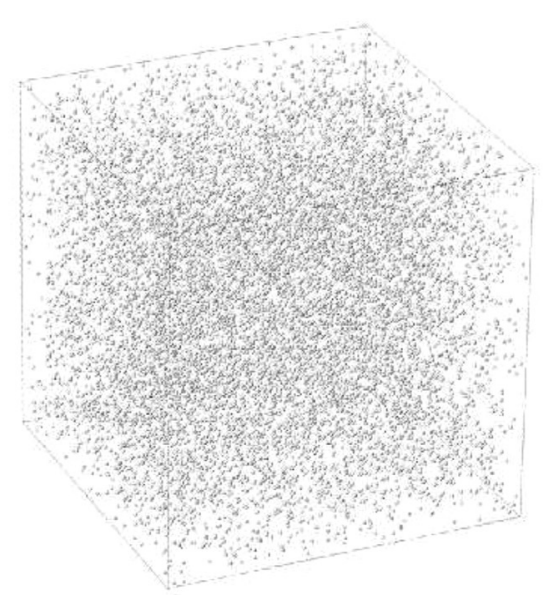

(a)

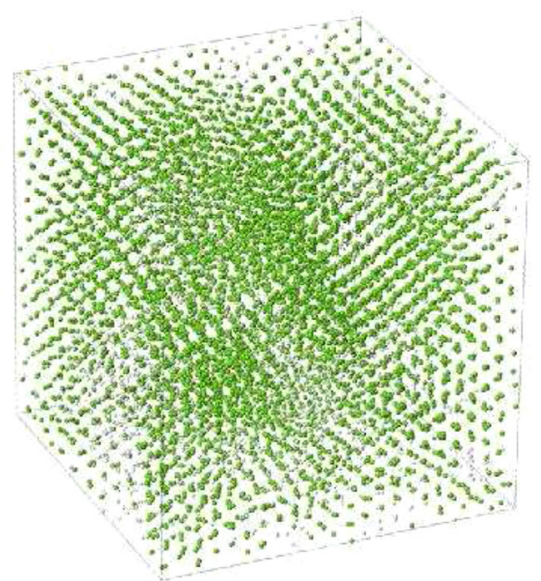

(c)

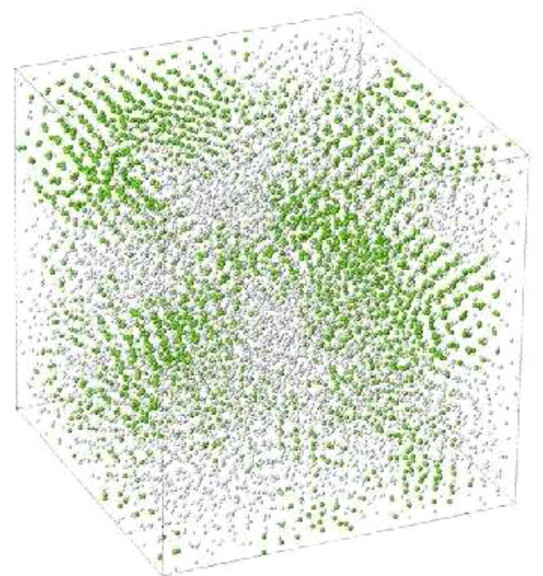

(b)

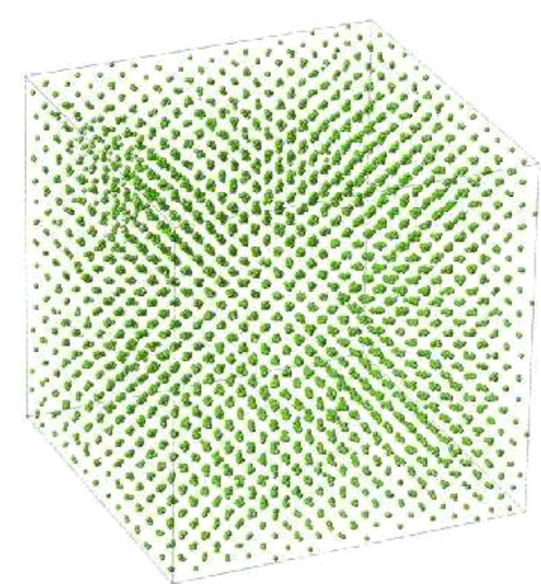

(d)

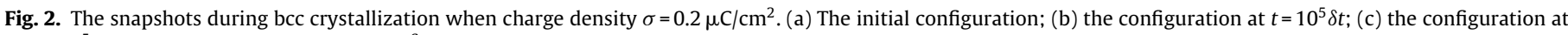
$t=2 \times 10^{5} \delta t ;(\mathrm{d})$ final configuration $\left(t=2 \times 10^{6} \delta t\right)$.

been reported long time ago that improper choice of particle number may mislead the resulted crystal structure due to the restriction of periodic boundary condition, especially when the simulation box is not large enough $[30,31,23]$. Under small system size, the particles are more readily to reach metastable bcc structures because there is a lower free-energy barrier for the formation of bcc crystallites from the melt, even though fcc is presumably the stable solid phase $[25,29]$.

Continuing to increase the charge density $\sigma$, a homogeneous to inhomogeneous transition will happen beyond a critical charge density. In a typical inhomogeneous phase, the stable voids are coexisting with ordered structures. Such phase transition has been observed both in experiment and in MC simulations, which is suggested to prove the existence of a long-range attraction in the interparticle interaction [32]. Fig. 4 shows the curves of RDF and $f_{\text {crystal }}$ during the dynamics process of void formation at charge density $\sigma=0.5 \mu \mathrm{C} / \mathrm{cm}^{2}$. In the top panel of Fig. 4, four curves of RDF at $10^{3} \delta t, 10^{4} \delta t, 10^{5} \delta t$ and $2 \times 10^{6} \delta t$ are given, respectively. The height of the first peak in RDF curve continues increasing with time, until it reaches a comparatively steady value after system's equilibrium. Some other peaks which indicates the fcc crystal structure also appear with the time. Note that the position of the first peak in RDF curves is smaller than that of perfect fcc crystal, because of the void formation. In the Bottom panel of Fig. 4, it shows the degree of crystallization $f_{\text {crystal }}$ varying with time step. $f_{\text {crystal }}$ increase apparently since the simulation starts until it also reaches a plateau after about $5 \times 10^{5} \delta$ t. The value of the plateau in $f_{\text {cryatal }}$ curve is even smaller than 0.8 , so there seems to be still some particles left not crystallized. Actually, most of such particles are near the boundary of the voids and not considered to be solid-like. Some snapshots of the system during the dynamic process of void formation can be seen in Fig. 5, corresponding to the RDF curves of Fig. 4. The growth of voids and the crystallization of colloids proceed simultaneously and they both keep stable after equilibrium. Unlike the results of previous MC simulations (only one or two voids formed probably due to the small size of system), there are several voids formed in the colloidal system. In experimental observations, the system that behaves as voids coexisting with order or disorder regions shows a spongy structure $[10,11,33]$. From this point of view, our results of MD simulations with much bigger system sizes are more likely to be in agreement with experimental results.

The average interparticle separation $D_{s}$ can be obtained from the position of the first peak in RDF curve. For perfect lattice, the average interparticle separation is defined as $D_{0}$. In reduced units of our simulations, $D_{0} \approx 0.879$ for perfect bcc and $D_{0} \approx 0.904$ for perfect fcc. The ratio $D_{s} / D_{0}$ is used to determine whether the ordered colloidal suspensions in the system are homogeneous or not: When $D_{s} / D_{0}=1$, the colloidal particles occupy the full volume and the suspensions exhibit a homogeneous phase; When $D_{s} / D_{0}<1$, it suggests that the suspensions could be inhomogeneous. Furthermore, we 

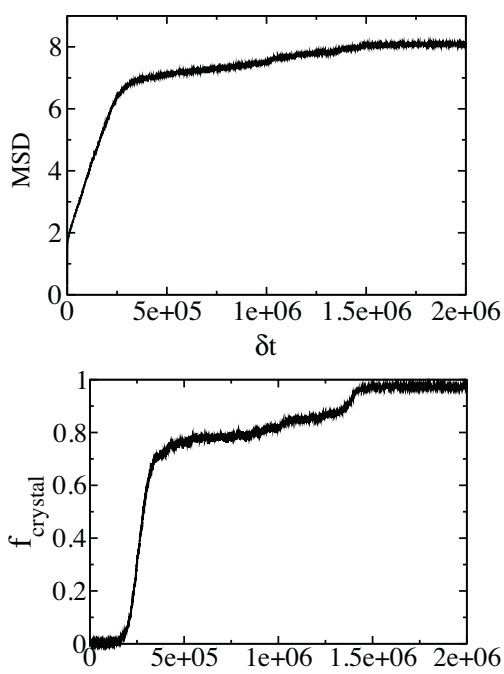

$\delta \mathrm{t}$

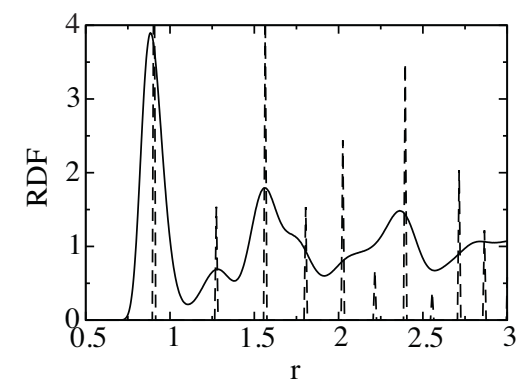

Fig. 3. From top to bottom: mean square distance (MSD) versus time step, the fraction of crystallization versus time step, averaged radial distribution function (RDF) after equilibrium. The dashed lines in RDF curve show the peaks of perfect fcc structure. The charge density is $\sigma=0.3 \mu \mathrm{C} / \mathrm{cm}^{2}$.
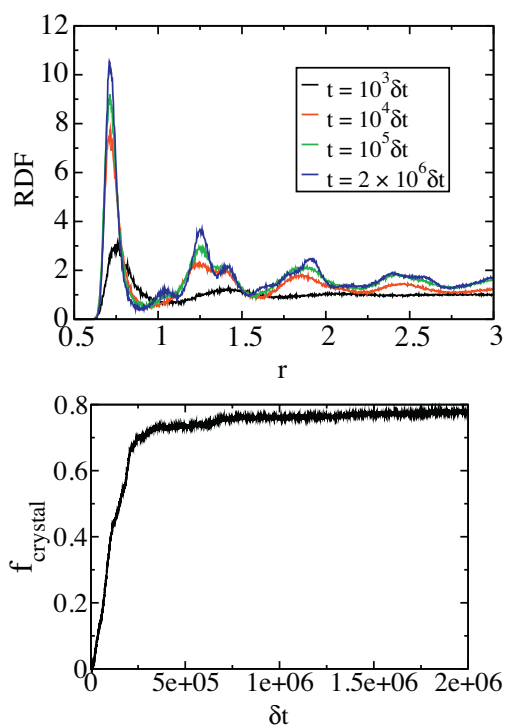

Fig. 4. Top: radial distribution function (RDF) at four typical time steps $10^{3} \delta t$ (black line), $10^{4} \delta t$ (red line), $10^{5} \delta t$ (green line), $2 \times 10^{6} \delta t$ (blue line). Bottom: the fraction of crystallization versus time step. The charge density is $\sigma=0.5 \mu \mathrm{C} / \mathrm{cm}^{2}$. (For interpretation of the references to color in this figure legend, the reader is referred to the web version of the article.) can estimate the fraction of the volume occupied by the voids using the ratio $D_{s} / D_{0}$, i.e,

$f_{\text {void }}=1-\left(\frac{D_{s}}{D_{0}}\right)^{3}$.

In Fig. 6, the ratio $D_{s} / D_{0}$ calculated as a function of charge density $\sigma$ is shown. As mentioned already, the system is found to have a homogeneous order firstly, as $D_{s} / D_{0}=1.0$. Continuing to increase $\sigma$, the system become inhomogeneous as $D_{S} / D_{0}<1.0$. From the curve of $D_{s} / D_{0}$, we can see that the homogeneous to inhomogeneous phase transition where $D_{s} / D_{0}$ drops is close to $\sigma=0.3 \mu \mathrm{C} / \mathrm{cm}^{2}$ (exactly between $\sigma=0.3 \mu \mathrm{C} / \mathrm{cm}^{2}$ and $\sigma=0.35 \mu \mathrm{C} / \mathrm{cm}^{2}$ ). This is a little different from the results of MC simulations [17], where homogeneous to inhomogeneous phase transition happened approximately at $\sigma=0.37 \mu \mathrm{C} / \mathrm{cm}^{2}$. According to Eq. (1), $R_{m}$ is also an important factor to explain the phase behaviors of the system such as the void formation and homogeneous to inhomogeneous transition. When the separation of interparticle $r_{i j}$ is less than $R_{m}$, the repulsive interaction is dominant to make the system show a homogenous state, otherwise the attractive interaction is dominant and the system is likely to become inhomogeneous. In this research, $R_{m}$ depends only on $\sigma$ and decreases with the increasing of $\sigma$ (see Eq. (4) and Fig. 6). Here we may assume that $R_{m}=D_{0}$ $\left(D_{0}=0.904\right.$, for the crystal structure in inhomogeneous phase is fcc), i.e, $R_{m} / D_{0}=1.0$ when the homogeneous to inhomogeneous transition begins to appear, so the corresponding value of $\sigma$ can be obtained based on Eq. (6) and $R_{m}=0.904$. Using this method, the critical value of $\sigma$ for homogeneous to inhomogeneous transition is solved to be $\sigma_{c} \approx 0.309 \mu \mathrm{C} / \mathrm{cm}^{2}$. In inhomogeneous region, the values of $R_{m}$ are always a little bit larger than $D_{s}$ (see Fig. (6)). Away from $\sigma_{c}$, the curve of $D_{s} / R_{m}$ are almost a plateau (the value of plateau is about 0.96 , see the inner panel of Fig. 6 ). From this, we can easily estimate the values of interparticle separation $D_{s}$ using calculated $R_{m}$ via Eq. (4) in inhomogeneous region especially away from the critical point, while the values of $D_{s}$ in homogeneous region are constant $D_{s}=D_{0}$.

In order to have a further study of the finite size effect on homogeneous to inhomogeneous phase transition, the results of $D_{S} / D_{0}$ obtained by MD simulations in the system with the same number of particles $N=432$ as that used in MC simulations, together with the results in the system with larger number of particles $N=10,976$, are listed in Table 1 . For the case $N=432$, the crystal structure becomes fcc until $\sigma \geq 0.4 \mu \mathrm{C} / \mathrm{cm}^{2}$, i.e, the bcc-fcc phase transition happens after the homogeneous to inhomogeneous phase transition, different from the results of $N=10,976$. Then we can estimate the critical value to get $\sigma_{c}=0.33 \mu \mathrm{C} / \mathrm{cm}^{2}$ for the homogeneous to inhomogeneous phase transition (Note that $D_{0}$ of perfect bcc is smaller than that of perfect fcc, so as to make a small shift of $\sigma_{c}$ ). As is shown in Table 1 , the finite size effects $\left(D_{s} / D_{0}\right)$ is significant near the critical point $\sigma_{c}$. But the finite size effects on $D_{s} / D_{0}$ become almost negligible when the charge density $\sigma$ is increased far away from the critical point.

In previous MC simulations, a glasslike inhomogeneous phase is observed as increasing the charge density $\sigma$ up to $0.68 \mu \mathrm{C} / \mathrm{cm}^{2}$ [17]. Later experiments on charged colloidal suspensions seems to confirm this by showing voids coexisting with dense amorphous regions [33]. Such agreement between experiments and simulations is considered to be a proof that SI potential can explain the reentrant phase transition of charge stabilized colloidal dispersions by varying the charge density $\sigma[32,34]$. As mentioned already, the number of particles used in MC simulations is only $N=432$. We have performed MD simulations using much larger number of particles $N=10,976$ at the same charge density $\sigma=0.68 \mu \mathrm{C} / \mathrm{cm}^{2}$ as MC simulations, but do not find any glasslike structures formation. The final configuration of the system at equilibrium is still the voids coexisting crystallites. Until the charge density $\sigma$ is increased to 


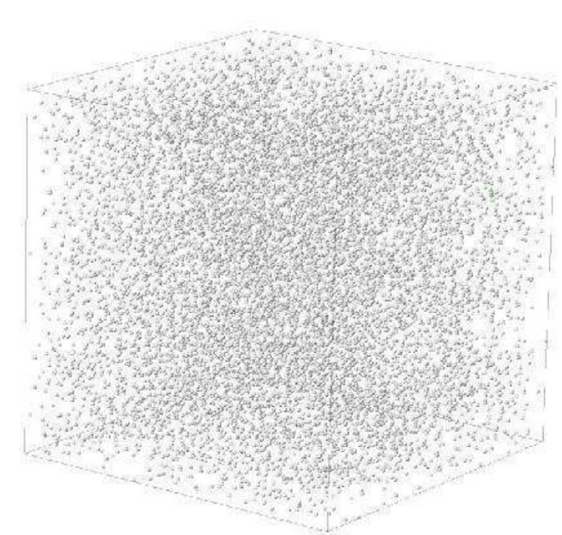

(a)

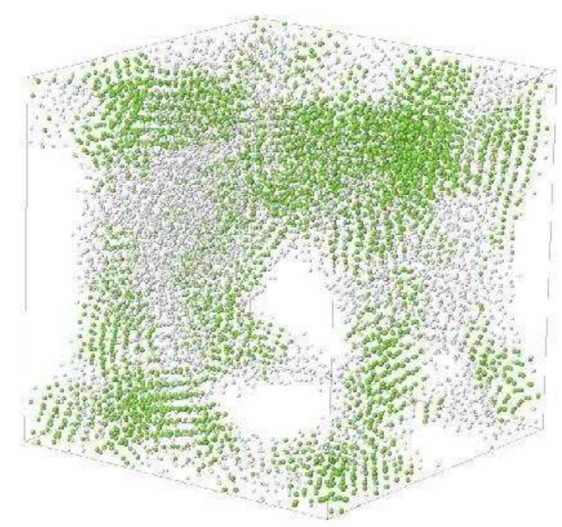

(c)

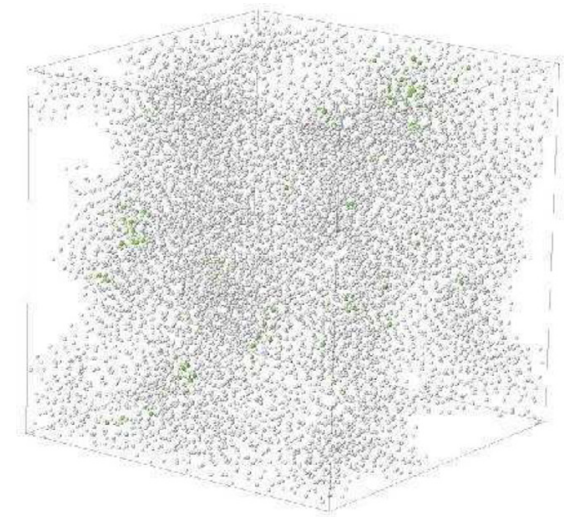

(b)

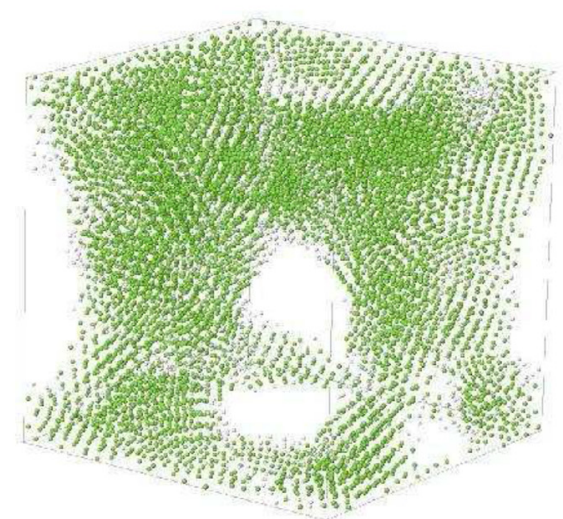

(d)

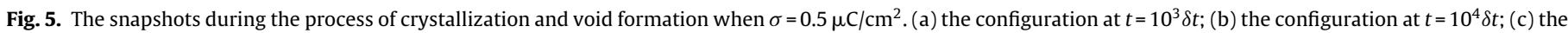
configuration at $t=10^{5} \delta t$; (d) final configuration $\left(t=2 \times 10^{6} \delta t\right)$.

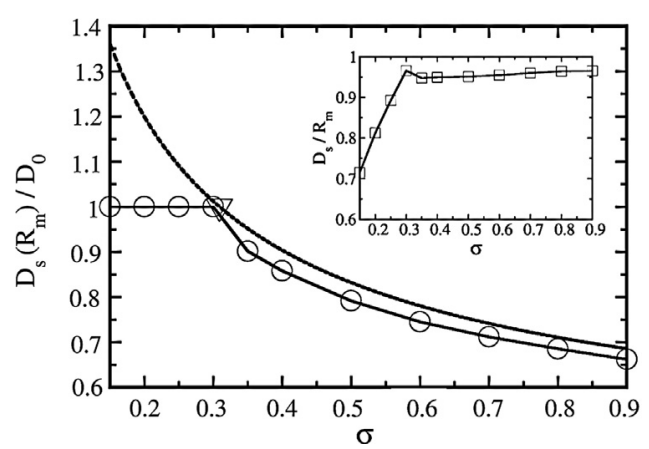

Fig. 6. Homogeneous to inhomogeneous phase transition with increasing the charge density $\sigma$. Circles+line: $D_{s} / D_{0}$; dashed line: $R_{m} / D_{0}$; triangles: critical point for homogeneous to inhomogeneous transition. The inset shows the curve of $D_{s} / R_{m}$ as a function of $\sigma$.

$\sigma=0.9 \mu \mathrm{C} / \mathrm{cm}^{2}$ which is even much larger than the value of the charge density used in MC simulations, the glasslike structures do not appear yet. Fig. 7 shows the curve of RDF and the snapshot of the system at $\sigma=0.9 \mu \mathrm{C} / \mathrm{cm}^{2}$. It can be seen obviously that the system exhibits an inhomogeneous phase with voids and crystallites, which is similar to the case of lower charge density, e.g. the system at $\sigma=0.5 \mu \mathrm{C} / \mathrm{cm}^{2}$ (see Figs. 4 and 5). The disagreement between previous MC simulations and our MD simulations is probably caused by the finite size effects too, i.e, the number of particles used in MC simulations is too small. As is shown in Fig. 6, the values of $D_{S} / D_{0}$ decrease with increasing of $\sigma$, so the volume occupied by the voids at higher charge density is larger resulting in more particles near the boundary of voids. In this situation, it is no wonder that the left particles for the crystallization is extremely few at very high charge density so that the nucleation is nearly impossible, if the number of particles used in simulations is small. In Fig. 8, we give the curve of averaged RDF and a snapshot of the system when equilibrium at very charge density $\sigma=0.9 \mu \mathrm{C} / \mathrm{cm}^{2}$ for the case $N=432$. Although RDF curve displays some peaks corresponding to fcc crystal structure, the peaks are lower than the case of $N=10,976$. Furthermore, the system does not exhibit any crystallization structures, because there are very few solid-like particles seen from the picture of the snapshot. Thus it may possibly lead to an artifact when performing simulations of the system with small number of particles at high charge density, although it does not

Table 1

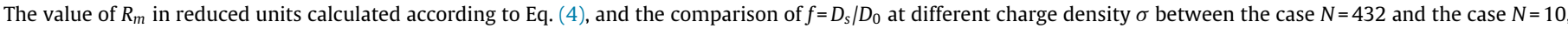

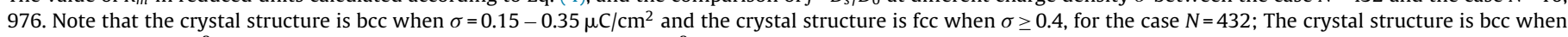
$\sigma=0.15-0.25 \mu \mathrm{C} / \mathrm{cm}^{2}$ and the crystal structure is fcc when $\sigma \geq 0.3 \mu \mathrm{C} / \mathrm{cm}^{2}$, for the case $N=10,976$.

\begin{tabular}{|c|c|c|c|c|c|c|c|c|c|c|c|}
\hline & 0.15 & 0.2 & 0.25 & 0.3 & 0.35 & 0.4 & 0.5 & 0.6 & 0.7 & 0.8 & 0.9 \\
\hline$R_{m}$ & 1.2302 & 1.084 & 0.9859 & 0.9147 & 0.8602 & 0.8169 & 0.7521 & 0.7056 & 0.6703 & 0.6425 & 0.6199 \\
\hline$f_{N=432}$ & 1.0 & 1.0 & 1.0 & 1.0 & 0.9461 & 0.8706 & 0.7981 & 0.7517 & 0.7152 & 0.6853 & 0.6654 \\
\hline$f_{N=10,976}$ & 1.0 & 1.0 & 1.0 & 1.0 & 0.9015 & 0.8580 & 0.7915 & 0.7450 & 0.7118 & 0.6853 & 0.6621 \\
\hline
\end{tabular}



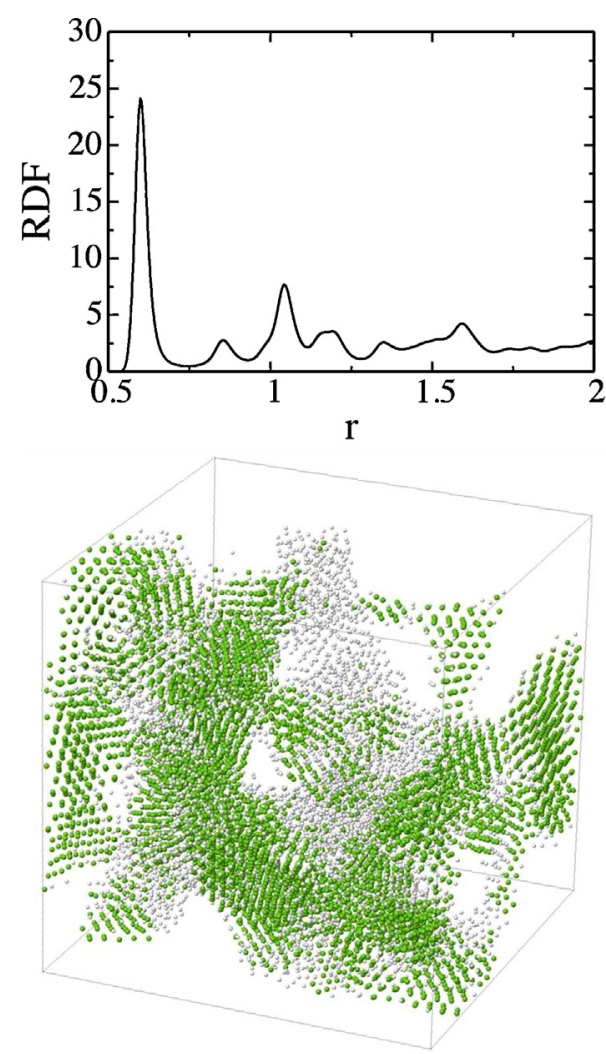

Fig. 7. The curve of averaged radial distribution function (RDF) and the final configuration of the system with $N=10,976$. The charge density is $\sigma=0.9 \mu \mathrm{C} / \mathrm{cm}^{2}$.
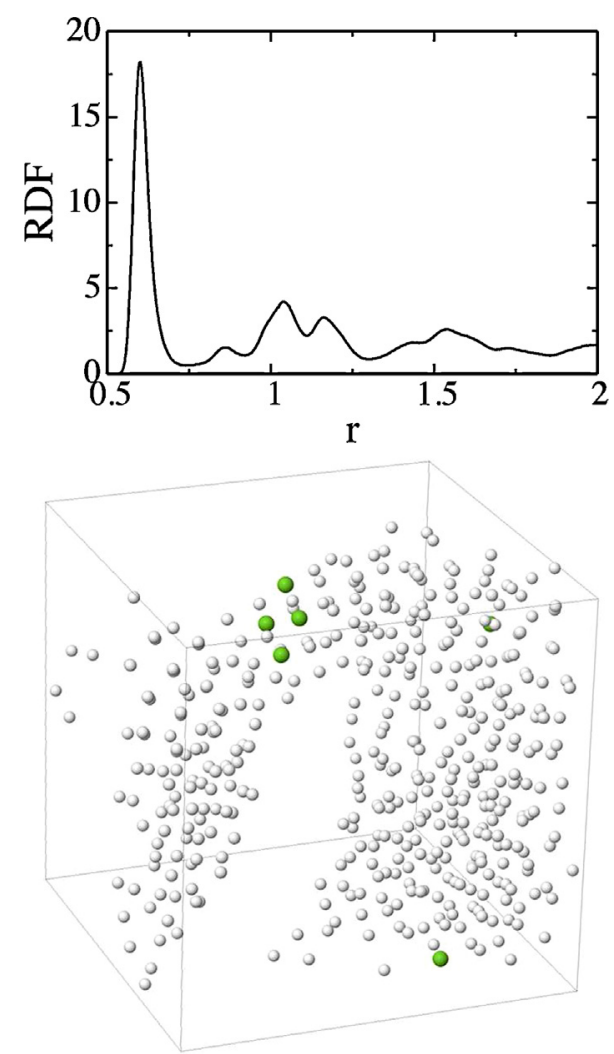

Fig. 8. The curve of averaged radial distribution function (RDF) and the final configuration after equilibrium of the system with $N=432$. The charge density is $\sigma=0.9 \mu \mathrm{C} / \mathrm{cm}^{2}$. affect the values of $D_{s} / D_{0}$ within statistical error. By the way, it has been discovered very earlier that the system size appears to have a large effect on the rate of nucleation. For instance, Honeycutt and Andersen have found that the size of the critical nucleus and the time of formation of a critical nucleus in Lennard-Jones system are smaller in a small system than those in a large system [23], indicating that the small system size and periodic boundary conditions are producing artifacts in the crystallization process.

\section{Conclusion}

We have performed MD simulations using sufficiently large number of particles $N=10,976$ to investigate the process of the structural ordering and voids formation in the charge stabilized colloidal suspensions. SI potential which has a long-range attraction is used to represent the interaction between colloidal particles. In this research, the influence of charge density on the phase behaviors of the colloidal system is studied in detail. Increasing the charge density, the colloidal system firstly begins to crystallize from liquid state and has a liquid-solid transition. Continuing to increase the charge density, the structural order of the system will have a bcc-fcc transition. Beyond a critical charge density the interparticle distance becomes smaller than that expected for a homogeneous dispersion and voids appear in the bulk of the suspension, i.e, the system exhibits a homogeneous to inhomogeneous transition. Such homogeneous to inhomogeneous transition is considered to be caused by the long-range attractions between particles. All of those results mentioned above are in agreement with previous observations of MC simulations and experiments, except that there is a shifting of the bcc-fcc boundary probably due to finite size effects.

The same number of particles as previous MC simulations is also used to carry out MD simulations and the finite size effects are studied. Besides the shifting of bcc-fcc boundary, we have found that the system with large number of particles $N=10,976$ at equilibrium is still the voids coexisting crystallites instead of a glasslike inhomogeneous phase observed in previous MC simulations and some experiments, when the charge density is increased to a very high value. In system with small sizes where $N=432$, MD simulations shows very few crystallized particles, which is in agreement with results of MC simulations, indicating that the glasslike or disordered phase region obtained at very high charge density in system of $N=432$ is probably an artifact produced by small system size and periodic boundary conditions. However, the glasslike or disordered phase coexisting with voids is indeed investigated in some experiments [10,33], so it seems that the results of our simulations are not in agreement with experimental observations. There may be possibly some reasons listed here: (1) The colloidal particles used in experiments are fairy large, and the relatively low diffusibility of such large particles make the system to cost a long time to reach the equilibrium, so the ordered structures could not form within the observation period. (2) The effect of polydispersity. In contrast to a single-component atomic system, the colloidal particles, even in a carefully prepared suspension, are usually not identical in size and charge density. The variations of size and charge from particle to particle can greatly influence the phase behaviors of the colloidal suspension. For instance, some studies have shown that beyond a critical polydispersity the colloidal crystals spontaneously transform to an amorphous structure [32,35]. (3) The effect of many-body interaction. It has been demonstrated that manybody interaction between particles does exist and has an effect on the liquid-solid phase transition of the charge stabilized colloidal suspensions [36,37]. Especially, the many-body interaction appears to be more significant in strongly interacting systems when the charge density is very high. At least, we can expect that the effective 
interaction between particles in homogeneous region is different from that in inhomogeneous regions.

In summary, the SI potential is a robust and impressive theory. It can be able to describe qualitatively the outcome of a wide range of experiments in colloidal suspensions, such as the structural ordering and void formation. However, SI theory should not be looked at as the final word to tell the whole story. From the data obtained in our MD simulations with sufficiently large number of particles (typically $N=10,976$ ) at high surface charge density, we may reasonably conclude that SI potential is not applicable for explaining the reentrant transition of highly charged colloidal systems $[12,13,38]$, and further studies (some other factors which influence the phase behavior of colloidal suspensions, e.g. the effect of polydispersity and many-body interaction, may be considered) are needed to be done in both experimental and theoretical aspects.

\section{Acknowledgments}

This work is supported by Grants 11172302, 20903112, 11302226,10972217 , and 11032011 from the National Natural Science Foundation of China.

\section{References}

[1] D. Frenkel, Colloidal systems - playing tricks with designer "atoms", Science 296 (2002) 65-66

[2] V.J. Anderson, H.N.W. Lekkerkerker, Insights into phase transition kinetics from colloid science, Nature 416 (2002) 811-815.

[3] W.B. Russel, Condensed-matter physics: tunable colloidal crystals, Nature 421 (2003) 490-491

[4] W. Poon, Colloids as big atoms, Science 304 (2004) 830-831.

[5] P. Schall, I. Cohen, D.A. Weitz, F. Spaepen, Visualization of dislocation dynamics in colloidal crystals, Science 305 (2004) 1944-1948.

[6] S.H. Xu, H.W. Zhou, Z.W. Sun, J.C. Xie, Formation of an fcc phase through a bcc metastable state in crystallization of charged colloidal particles, Phys. Rev. E 82 (2010) 010401.

[7] E.J.W. Verwey, J.T.G. Overbeek, Theory of the Stability of Lyophobic Colloids, Elsevier, New York, 1948.

[8] A.K. Arora, B.V.R. Tata, A.K. Sood, R. Kesavamoorthy, Reentrant phase-transition in charged colloidal suspensions, Phys. Rev. Lett. 60 (1988) 2438-2441.

[9] B.V.R. Tata, M. Rajalakshmi, A.K. Arora, Vapor-liquid condensation in charged colloidal suspensions, Phys. Rev. Lett. 69 (1992) 3778-3781.

[10] K. Ito, H. Yoshida, N. Ise, Void structure in colloidal dispersions, Science 263 (1994) 66-68.

[11] H. Yoshida, N. Ise, T. Hashimoto, Void structure and vapor-liquid condensation in dilute deionized colloidal dispersions, J. Chem. Phys. 103 (1995) 10146-10151

[12] J. Yamanaka, H. Yoshida, T. Koga, N. Ise, T. Hashimoto, Reentrant solid-liquid transition in ionic colloidal dispersions by varying particle charge density, Phys. Rev. Lett. 80 (1998) 5806-5809.

[13] H. Yoshida, J. Yamanaka, T. Koga, T. Koga, N. Ise, T. Hashimoto, Transitions between ordered and disordered phases and their coexistence in dilute ionic colloidal dispersions, Langmuir 15 (1999) 2684-2702.

[14] B.V.R. Tata, S.S. Jena, Ordering, dynamics and phase transitions in charged colloids, Solid. State. Commun 139 (2006) 562-580.
[15] I. Sogami, N. Ise, On the electrostatic interaction in macroionic solutions, J. Chem. Phys. 81 (1984) 6320-6332.

[16] B.V.R. Tata, A.K. Arora, M.C. Valsakumar, Reentrant phase-transition in charged colloidal suspensions - a Monte-Carlo study, Phys. Rev. E 47 (1993) $3404-$ 3411.

[17] B.V.R. Tata, N. Ise, Homogeneous to inhomogeneous transition in charged colloids, Phys. Rev. B 54 (1996) 6050-6053.

[18] B.V.R. Tata, N. Ise, Monte carlo study of structural ordering in charged colloids using a long-range attractive interaction, Phys. Rev. E 58 (1998) 2237-2246.

[19] W.H. Mulder, On the theory of electrostatic interactions in suspensions of charged colloids, Soil Sci. Soc. Am. J. 74 (2010) 1-4.

[20] R. van Roij, M. Dijkstra, J.P. Hansen, Phase diagram of charge-stabilized colloidal suspensions: van der waals instability without attractive forces, Phys. Rev. E 59 (1999) 2010-2025.

[21] R. van Roij, Attraction or repulsion between charged colloids? a connection with debye-huckel theory, J. Phys.: Condens. Matter 12 (2000) A263-A267.

[22] J.D. Honeycutt, H.C. Andersen, The effect of periodic boundary conditions on homogeneous nucleation observed in computer simulations, Chem. Phys. Lett. 108 (1984) 535-538.

[23] J.D. Honeycutt, H.C. Andersen, Small system size artifacts in the moleculardynamics simulation of homogeneous crystal nucleation in supercooled atomic liquids, J. Phys. Chem. 90 (1986) 1585-1589.

[24] W.C. Swope, H.C. Andersen, 10(6)-Particle molecular-dynamics study of homogeneous nucleation of crystals in a supercooled atomic liquid, Phys. Rev. B 41 (1990) 7042-7054.

[25] J.S. Vanduijneveldt, D. Frenkel, Computer-simulation study of free-energy barriers in crystal nucleation, J. Chem. Phys. 96 (1992) 4655-4668.

[26] M.P. Allen, D.J. Tildesley, Computer Simulation of Liquids, Clarendon Press, Oxford, 1987.

[27] D. Frenkel, B. Smit, Understanding Molecular Simulation: From Algorithms to Applications, 2nd ed., Academic Press, San Diego, 2001.

[28] H.J.C. Berendsen, J.P.M. Postma, W.F. Vangunsteren, A. Dinola, J.R. Haak, Molecular-dynamics with coupling to an external bath, J. Chem. Phys. 81 (1984) 3684-3690.

[29] P.R. tenWolde, M.J. RuizMontero, D. Frenkel, Numerical calculation of the rate of crystal nucleation in a Lennard-Jones system at moderate undercooling, J. Chem. Phys. 104 (1996) 9932-9947.

[30] M.J. Mandell, J.P. Mctague, A. Rahman, Crystal nucleation in 3-dimensional Lennard-Jones system. 2. Nucleation kinetics for 256 and 500 particles, J. Chem. Phys. 66 (1977) 3070-3075.

[31] J.N. Cape, J.L. Finney, L.V. Woodcock, An analysis of crystallization by homogeneous nucleation in a 4000-atom soft-sphere model, J. Chem. Phys. 75 (1981) $2366-2373$.

[32] A.K. Arora, B.V.R. Tata, Interactions, structural ordering and phase transitions in colloidal dispersions, Adv. Colloid Interface Sci. 78 (1998) 49-97.

[33] B.V.R. Tata, E. Yamahara, P.V. Rajamani, N. Ise, Amorphous clustering in highly charged dilute poly(chlorostyrene-styrene sulfonate) colloids, Phys. Rev. Lett. 78 (1997) 2660-2663.

[34] N. Ise, I.S. Sogami, Structure Formation in Solution: Ionic Polymers and Colloidal Particles, Springer-Verlag, Berlin, 2005.

[35] P.N. Pusey, E. Zaccarelli, C. Valeriani, E. Sanz, W.C.K. Poon, M.E. Cates, Hard spheres: crystallization and glass formation, Phil. Trans. R. Soc. A 367 (2009) 4993-5011.

[36] P.E. Dyshlovenko, Evidence of many-particle interactions in two-dimensional charge-stabilized colloidal crystals, Phys. Rev. Lett. 95 (2005) 038302.

[37] J. Dobnikar, R. Rzehak, H.H. von Grunberg, Effect of many-body interactions on the solid-liquid phase behavior of charge-stabilized colloidal suspensions, Europhys. Lett. 61 (2003) 695-701.

[38] P. Wette, I. Klassen, D. Holland-Moritz, D.M. Herlach, H.J. Schope, N. Lorenz, H. Reiber, T. Palberg, S.V. Roth, Communications: Complete description of reentrant phase behavior in a charge variable colloidal model system, J. Chem. Phys. 132 (2010) 131102. 